\title{
Pacific
}

Journal of

Mathematics

\section{HOMOGENEITY GROUPS OF ENDS OF OPEN 3-MANIFOLDS}

DENNIS J. GARITY AND DUŠAN REPOVŠ 


\title{
HOMOGENEITY GROUPS OF ENDS OF OPEN 3-MANIFOLDS
}

\author{
DENNIS J. GARITY AND DUŠAN REPOVŠ
}

\begin{abstract}
For every finitely generated abelian group $G$, we construct an irreducible open 3-manifold $M_{G}$ whose end set is homeomorphic to a Cantor set and whose homogeneity group is isomorphic to $G$. The end homogeneity group is the group of self-homeomorphisms of the end set that extend to homeomorphisms of the 3-manifold. The techniques involve computing the embedding homogeneity groups of carefully constructed Antoine-type Cantor sets made up of rigid pieces. In addition, a generalization of an Antoine Cantor set using infinite chains is needed to construct an example with integer homogeneity group. Results about the local genus of points in Cantor sets and about the geometric index are also used.
\end{abstract}

\section{Introduction}

Each Cantor set $C$ in $S^{3}$ has for complement an open 3-manifold $M^{3}$ with end set $C$. Properties of the embedding of the Cantor set give rise to properties of the corresponding complementary 3-manifold $M^{3}$. See [Souto and Stover 2013], [Garity and Repovš 2013], and [Garity et al. 2014] for examples of this.

We investigate possible group actions on the end set $C$ of the open 3-manifold $M^{3}$ in the following sense: the homogeneity group of the end set is the group of homeomorphisms of the end set $C$ that extend to homeomorphisms of the open 3-manifold $M^{3}$. Referring specifically to the embedding of the Cantor set, this group can also be called the embedding homogeneity group of the Cantor set. See [Dijkstra 2010] and [van Mill 2011] for some other types of homogeneity.

The standardly embedded Cantor set is at one extreme here. The embedding homogeneity group is the full group of self-homeomorphisms of the Cantor set, an extremely rich group (there is such a homeomorphism taking any countable dense set to any other). Cantor sets with this full embedding homogeneity group are called strongly homogeneously embedded. See [Daverman 1979] for an example of a nonstandard Cantor set with this property.

At the other extreme are rigidly embedded Cantor sets, those Cantor sets for

MSC2010: primary 54E45, 57M30, 57N12; secondary 57N10, 54F65.

Keywords: open 3-manifold, rigidity, manifold end, geometric index, Cantor set, homogeneity group, abelian group, defining sequence. 
which only the identity homeomorphism extends. Shilepsky [1974] constructed Antoine-type [1920] rigid Cantor sets. Their rigidity is a consequence of Sher's result [1968] that if two Antoine Cantor sets are equivalently embedded, the stages of their defining sequences must match up exactly. In the last decade, new examples [Garity et al. 2006; 2011] of nonstandard Cantor sets were constructed that were both rigidly embedded and had simply connected complement. See [Wright 1986] for additional examples of rigidity.

These examples naturally lead to the question of which types of groups can arise as end homogeneity groups between the two extremes mentioned above. In this paper we show that for each finitely generated abelian group $G$, there is an irreducible open 3-manifold with end set homeomorphic to a Cantor set and end homogeneity group isomorphic to $G$. (See Corollary 6.3.)

The Cantor sets produced are unsplittable, in the sense that for each such $C$, no 2-sphere in the complement of $C$ separates points of $C$. We produce these examples by constructing, for each natural number $m$ greater than one, 3-manifolds with end homogeneity groups $\mathbb{Z}_{m}$, and by separately constructing 3 -manifolds with end homogeneity group $\mathbb{Z}$. We then link the Cantor sets needed for a given abelian group in an unsplittable manner.

In Section 2, we give definitions and the basic results needed for the rest of the paper. In Section 3, we review the needed results about Antoine Cantor sets. In Section 4 we produce Cantor sets with embedding homogeneity group $\mathbb{Z}_{m}$. In Section 5 we produce Cantor sets with embedding homogeneity group $\mathbb{Z}$. Section 6 ties together the previous results and lists and proves the main theorems. Section 7 lists some remaining questions.

\section{Preliminaries}

Background. Refer to [Garity et al. 2005; 2006; 2014] for a discussion of Cantor sets in general and of rigid Cantor sets, and to [Željko 2005] for results about the local genus of points in Cantor sets and defining sequences for Cantor sets. The bibliographies in these papers contain additional references to results about Cantor sets. Two Cantor sets $X$ and $Y$ in $S^{3}$ are said to be equivalent if there is a self-homeomorphism of $S^{3}$ taking $X$ to $Y$; otherwise they are inequivalent, or inequivalently embedded. A Cantor set $C$ is rigidly embedded in $S^{3}$ if the only self-homeomorphism of $C$ that extends to a homeomorphism of $S^{3}$ is the identity.

Geometric index. We list the results we need on geometric index. See [Schubert 1953] and [Garity et al. 2011] for more details.

If $K$ is a link in the interior of a solid torus $T$, the geometric index of $K$ in $T$, denoted by $\mathrm{N}(K, T)$, is defined as the minimum of $|K \cap D|$ over all meridional disks $D$ of $T$ intersecting $K$ transversely. If $T$ is a solid torus and $M$ is a finite 
union of disjoint solid tori such that $M \subset \operatorname{Int}(T)$, then the geometric index $\mathrm{N}(M, T)$ of $M$ in $T$ is $\mathrm{N}(K, T)$, where $K$ is a core of $M$.

Theorem 2.1 [Schubert 1953; Garity et al. 2011, Theorem 3.1]. Let $T_{0}$ and $T_{1}$ be unknotted solid tori in $S^{3}$ with $T_{0} \subset \operatorname{Int}\left(T_{1}\right)$ and $\mathrm{N}\left(T_{0}, T_{1}\right)=1$. Then $\partial T_{0}$ and $\partial T_{1}$ are parallel; i.e., the manifold $T_{1}-\operatorname{Int}\left(T_{0}\right)$ is homeomorphic to $\partial T_{0} \times I$, where $I$ is the closed unit interval $[0,1]$.

Theorem 2.2 [Schubert 1953; Garity et al. 2011, Theorem 3.2]. Let $T_{0}$ be a finite union of disjoint solid tori. Let $T_{1}$ and $T_{2}$ be solid tori such that $T_{0} \subset \operatorname{Int}\left(T_{1}\right)$ and $T_{1} \subset \operatorname{Int}\left(T_{2}\right)$. Then $\mathrm{N}\left(T_{0}, T_{2}\right)=\mathrm{N}\left(T_{0}, T_{1}\right) \cdot \mathrm{N}\left(T_{1}, T_{2}\right)$.

There is one additional result we will need:

Theorem 2.3 [Schubert 1953; Garity et al. 2011, Theorem 3.3]. Let T be a solid torus in $S^{3}$ and let $T_{1}, \ldots, T_{n}$ be unknotted pairwise disjoint solid tori in $T$, each of geometric index 0 in $T$. Then the geometric index of $\bigcup_{i=1}^{n} T_{i}$ in $T$ is even.

Defining sequences and local genus. We review the definition and some basic facts from [Željko 2005] about the local genus of points in a Cantor set. See that work for a discussion of defining sequences.

Let $\mathscr{D}(X)$ be the set of all defining sequences for a Cantor set $X \subset S^{3}$. Let $\left(M_{i}\right) \in \mathscr{D}(X)$ be a specific defining sequence for an $X$. For $A \subset X$, denote by $M_{i}^{A}$ the union of those components of $M_{i}$ which intersect $A$. The genus $g\left(M_{i}^{A}\right)$ of $M_{i}^{A}$ is the maximum genus of a component of $M_{i}^{A}$. Define

$g_{A}\left(X ;\left(M_{i}\right)\right)=\sup \left\{g\left(M_{i}^{A}\right): i \geq 0\right\}, \quad g_{A}(X)=\inf \left\{g_{A}\left(X ;\left(M_{i}\right)\right):\left(M_{i}\right) \in \mathscr{D}(X)\right\}$.

The number $g_{A}(X)$ is called the genus of the Cantor set $X$ with respect to the subset $A$. For $A=\{x\}$ we call the number $g_{\{x\}}(X)$ the local genus of the Cantor set $X$ at the point $x$ and denote it by $g_{x}(X)$.

Let $x$ be an arbitrary point of a Cantor set $X$ and $h: S^{3} \rightarrow S^{3}$ a homeomorphism. Then the local genus $g_{x}(X)$ is the same as the local genus $g_{h(x)}(h(X))$. Also note that if $x \in C \subset C^{\prime}$, then the local genus of $x$ in $C$ is less than or equal to the local genus of $x$ in $C^{\prime}$. See [Željko 2005, Theorem 2.4].

The following result is needed to show that certain points in our examples have local genus 2 .

Theorem 2.4 [Željko 2005]. Let $X, Y \subset S^{3}$ be Cantor sets and $p \in X \cap Y$. Suppose there exists $a$ 3-ball $B$ and $a$ 2-disk $D \subset B$ such that

(1) $p \in \operatorname{Int}(B), \partial D=D \cap \partial B, D \cap(X \cup Y)=\{p\}$; and

(2) $X \cap B \subset B_{X} \cup\{p\}$ and $Y \cap B \subset B_{Y} \cup\{p\}$, where $B_{X}$ and $B_{Y}$ are the components of $B-D$.

Then $g_{p}(X \cup Y)=g_{p}(X)+g_{p}(Y)$. 
Discussion and examples of ends and homogeneity groups. For background on Freudenthal compactifications and theory of ends, see [Dickman 1968; Freudenthal 1942; Siebenmann 1965]. For an alternate proof using defining sequences of the result that every homeomorphism of the open 3-manifold extends to a homeomorphism of its Freudenthal compactification, see [Garity and Repovš 2013].

At the end of the next section, we will discuss elements of the homogeneity group of a standard self-similar Antoine Cantor set. Note that removing $n$ points from $S^{3}$ yields a reducible open 3-manifold with end homogeneity group the symmetric group on $n$ elements. It is not immediately obvious how to produce examples that are irreducible, have a rich end structure (for example a Cantor set), and at the same time have specified abelian end homogeneity groups.

\section{Properties of the Antoine Cantor set}

An Antoine Cantor set is described by a defining sequence $\left(M_{i}\right)$ as follows: Let $M_{0}$ be an unknotted solid torus in $S^{3}$. Let $M_{1}$ be a chain of at least four linked, pairwise disjoint, unknotted solid tori in $M_{0}$, as in Figure 1. Inductively, $M_{i}$ consists of pairwise disjoint solid tori in $S^{3}$ and $M_{i+1}$ is obtained from $M_{i}$ by placing a chain of at least four linked, pairwise disjoint, unknotted solid tori in each component of $M_{i}$. If the diameter of the components goes to 0, the Antoine Cantor set is $C=\bigcap_{i=0}^{\infty} M_{i}$.

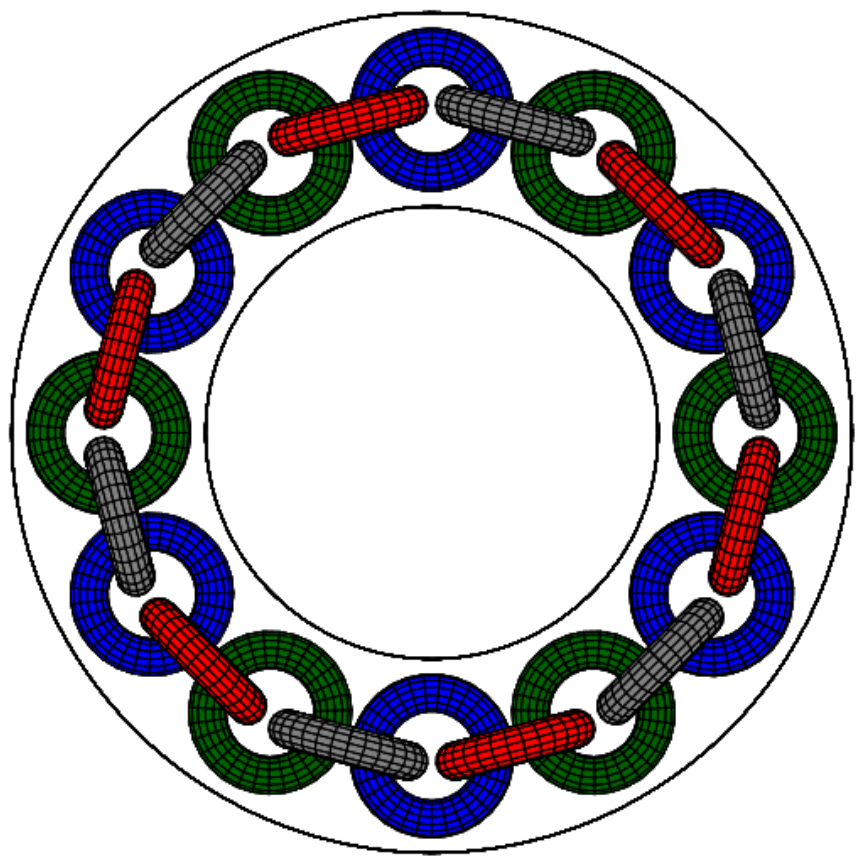

Figure 1. Antoine chain with $\mathbb{Z}_{6}$ group action. 
We refer to [Sher 1968] for basic results and description of Antoine Cantor sets. The key result we shall need is the following:

Theorem 3.1 [Sher 1968, Theorems 1 and 2]. Two Antoine Cantor sets in $S^{3}$, with defining sequences $\left(M_{i}\right)$ and $\left(N_{i}\right)$, respectively, are equivalently embedded if and only if there is a self-homeomorphism $h$ of $S^{3}$ with $h\left(M_{i}\right)=N_{i}$ for each $i$.

In particular, the number and adjacency of links in the chains must match up at each stage. Because we need a modification of this result for infinite chains in Section 5, we outline an alternative proof of the forward implication.

Proof of forward implication of Theorem 3.1. It suffices to show that if $C$ has two Antoine defining sequences $\left(M_{i}\right)$ and $\left(N_{i}\right)$, then there is a homeomorphism $h$ as in the theorem.

Step 1: There is a general position homeomorphism $h_{1}$, fixed on $C$, such that $h_{1}\left(\partial\left(M_{1}\right) \cup \partial\left(M_{2}\right)\right)$ is in general position with $\partial\left(N_{1}\right) \cup \partial\left(N_{2}\right)$. The curves of intersection of $h_{1}\left(\partial\left(M_{1}\right) \cup \partial\left(M_{2}\right)\right) \cap\left(\partial\left(N_{1}\right) \cup \partial\left(N_{2}\right)\right)$ can be eliminated by a homeomorphism $h_{2}$ also fixed on $C$, by a standard argument and the facts that any nontrivial curve on $\partial\left(M_{i}\right)$ does not bound a disk in the complement of $C$ and that no 2-sphere separates the points of $C$. For details on the type of argument in this step, see [Sher 1968] or [Garity et al. 2011].

Step 2: Let $T$ be a component of $h_{2} \circ h_{1}\left(M_{1}\right)$ and assume $T$ intersects a component $S$ of $N_{1}$. Either $T \subset \operatorname{Int}(S)$ or $S \subset \operatorname{Int}(T)$. First assume $T \subset \operatorname{Int}(S)$. If the geometric index of $T$ in $S$ is 0 , then since the other components of $h_{2} \circ h_{1}\left(M_{1}\right)$ are linked to $T$ by a finite chain, all components of $h_{2} \circ h_{1}\left(M_{1}\right)$ are in the interior of $S$. This is a contradiction since there are points of $C$ not in $S$. So the geometric index of $T$ in $S$ is greater than or equal to 1 .

Note that $T$ cannot be contained in any component of $N_{2}$ that is in $S$ since these have geometric index 0 in $S$. So $T$ contains all the components of $N_{2}$ that are in $S$. Each of these components has geometric index 0 in $T$, so the union of these components has an even geometric index in $T$ by Theorem 2.3. This geometric index must then be 2 and the geometric index of $T$ in $S$ must be 1. Now there is a homeomorphism $h_{3}$, fixed on $C$ and the complement of $S$, that takes $T$ to $S$.

If instead $S \subset \operatorname{Int}(T)$, a similar argument shows there is a homeomorphism $h_{3}$, fixed on $C$ and the complement of $T$, taking $S$ to $T$. The net result is that it possible to construct a homeomorphism $h_{3}^{\prime}$ taking the components of $h_{2} \circ h_{1}\left(M_{1}\right)$ to the components of $S$. One now proceeds inductively, matching up further stages in the constructions, obtaining the desired homeomorphism $h$ as a limit.

Remark 3.2. A standard argument shows that an Antoine Cantor set cannot be separated by a 2 -sphere. This is also true if the construction starts with a finite open chain of linked tori as in Figure 3. 
Remark 3.3. Also note that the homeomorphism of Theorem 3.1 can be realized as the final stage of an isotopy since each of the homeomorphisms in the argument can be realized by an isotopy.

Homogeneity groups of Antoine Cantor sets. Let $C$ be obtained by a standard Antoine construction where the same number of tori are used in tori of previous stages in each stage of the construction. For example, the Antoine pattern in Figure 1 with 24 smaller tori, each geometrically similar to the outer torus, can be repeated in each component at each stage of the construction.

We now consider some elements of the embedding homogeneity group of $C$. There is an obvious $\mathbb{Z}_{24}$ group action on the resulting Cantor set obtained by rotating and twisting the large torus. There is also a $\mathbb{Z}_{24} \oplus \mathbb{Z}_{24}$ action on $C$ obtained by considering the first two stages, where we require each torus in the second stage to rotate the same amount. If we allow the tori in the second stage to rotate different amounts, we get an even larger group action by a wreath product of $\mathbb{Z}_{24}$ with itself. Considering more stages results in even more complicated group actions.

In addition to these group actions arising from rotating and twisting, there are also orientation-reversing $\mathbb{Z}_{2}$ actions that arise from reflecting through a horizontal plane (containing the core of the large torus) or through a vertical plane (containing meridians of the large torus).

From this we see that even for a simple self-similar Antoine Cantor set, the embedding homogeneity group is more complex than just the group of obvious rotations from the linking structure. In the next section we shall carefully combine certain Antoine constructions to produce a more rigid example with nontrivial end homogeneity group, in such a way that these kinds of orientation-reversing homeomorphisms are not possible, and that also restricts the possible rotations.

\section{A Cantor set with embedding homogeneity group $\mathbb{Z}_{m}$}

Fix an integer $m>1$. We describe how to construct a Cantor set in $S^{3}$ with embedding homogeneity group $\mathbb{Z}_{m}$.

Construction 4.1. As in the previous section, let $S_{0}$ be an unknotted solid torus in $S^{3}$. Let $\left\{S_{(1, i)}: 1 \leq i \leq 4 m\right\}$ be an Antoine chain of $4 m$ pairwise disjoint linked solid tori in the interior of $S_{0}$ and let

$$
S_{1}=\bigcup_{i=1}^{4 m} S_{(1, i)} .
$$

See Figure 1 for the case when $m=6$. Let $C_{j}, 1 \leq j \leq 4$, be a rigid Antoine Cantor set with first stage $S_{(1, j)}$. Choose these four rigid Antoine Cantor sets so that they are inequivalently embedded in $S^{3}$. Let $h$ be a homeomorphism of $S^{3}$, 
fixed on the complement of the interior of $S_{0}$, that takes $S_{(1, j)}$ to $\left.S_{(1, j+4} \bmod 4 m\right)$ for $1 \leq j \leq 4 m$. Require that $h^{m}$ is the identity on each $S_{(1, i)}$.

For $4 k<i \leq 4 k+4$, let $C_{i}$ be the rigid Cantor set in $S_{(1, i)}$ given by $h^{k}\left(C_{i-4 k}\right)$. Note that this produces $m$ copies of each of the rigid Cantor sets $C_{1}, C_{2}, C_{3}$, and $C_{4}$. Again, see Figure 1, where the coloring indicates the four classes of rigid Cantor sets. The Cantor set we are looking for is

$$
C=\bigcup_{i=1}^{4 m} C_{i} .
$$

Theorem 4.2. The Cantor set $C$ from the previous construction has embedding homogeneity group $\mathbb{Z}_{m}$ and is unsplittable.

Proof. Let $\ell: S^{3} \rightarrow S^{3}$ be a homeomorphism taking $C$ to $C$. We show that $\left.\ell\right|_{C}=\left.h^{k}\right|_{C}$ for some $k, 1 \leq k \leq m$. By [Sher 1968], we may assume that $\ell$ takes each $S_{(1, i)}$ to some $S_{(1, j)}$, and so $\ell\left(C_{i}\right)=C_{j}$. Because of the distinct rigid Cantor sets involved, this is only possible if $j-i \equiv 0 \bmod 4$.

So assume that $\ell\left(S_{(1,1)}\right)=S_{(1,4 k+1)}$. Then $\ell\left(S_{(1,2)}\right)$ must be one of the two tori linked with $S_{(1,4 k+1)}$, namely $S_{(1,4 k)}$ or $S_{(1,4 k+2)}$. Since $(4 k-2) \not \equiv 0 \bmod 4, \ell\left(S_{(1,2)}\right)$ must be $S_{(1,4 k+2)}$. Continuing inductively, one sees that $\ell\left(S_{(1, i)}\right)=S_{(1,4 k+i \bmod 4 m)}$. Thus $\ell\left(C_{i}\right)=C_{4 k+i \bmod 4 m}$. But $h^{k}\left(C_{i}\right)$ is also $C_{4 k+i \bmod 4 m}$. Since these are rigid Cantor sets, $\left.\ell\right|_{C_{i}}=\left.h^{k}\right|_{C_{i}}$ for each $i$.

So the embedding homogeneity group of $C$ is $\left\{h^{k}: 1 \leq k \leq p\right\} \simeq \mathbb{Z}_{m}$. By Remark 3.2, $C$ is unsplittable. The assertion follows.

\section{A Cantor set with embedding homogeneity group $\mathbb{Z}$}

We now construct a Cantor set in $S^{3}$ with embedding homogeneity group $\mathbb{Z}$. This requires careful analysis of an infinite chain analogue of the Antoine construction.

Construction 5.1. Let $S_{0}$ be a pinched solid torus in $S^{3}$, i.e., the quotient of a solid torus with a meridional disk collapsed to a single point $w$. Let $T_{i}, i \in \mathbb{Z}$, be a countable collection of unknotted pairwise disjoint solid tori in $S_{0}$ such that each $T_{i}$ is of simple linking type with both $T_{i-1}$ and $T_{i+1}$, and is not linked with $T_{j}, j \neq i-1$ or $i+1$. Place the tori $T_{i}$ so that the $T_{i}, i>0$, and the $T_{i}, i<0$, have $w$ as a limit point as in Figure 2.

For $1 \leq j \leq 3$, let $C_{j}$ be a rigid Antoine Cantor set with first stage $T_{j}$. Choose these three rigid Antoine Cantor sets so that they are inequivalently embedded in $S^{3}$. Let $\alpha$ be a homeomorphism of $S^{3}$, fixed on the complement of the interior of $S_{0}$, that takes $T_{j}$ to $T_{j+3}$ for $j \in \mathbb{Z}$.

For $3 k<i \leq 3 k+3$, let $C_{i}$ be the rigid Cantor set in $T_{i}$ given by $\alpha^{k}\left(C_{i-3 k}\right)$. Note that this produces a countable number of copies of each of the rigid Cantor 


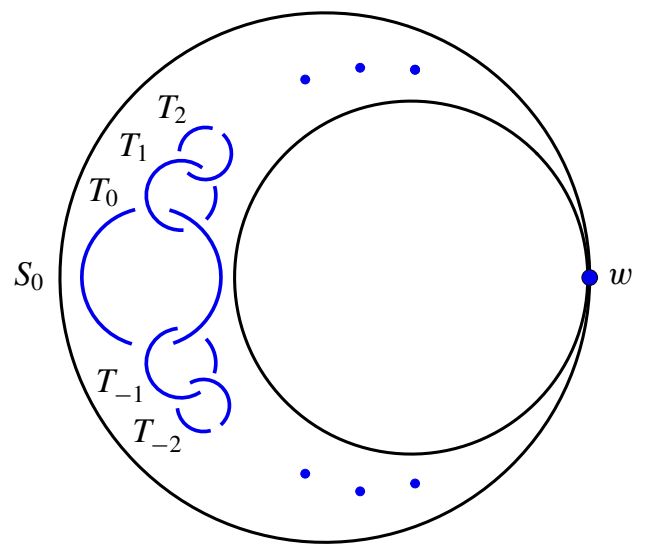

Figure 2. Infinite Antoine chain.

sets $C_{1}, C_{2}$, and $C_{3}$. Again, see Figure 2. The Cantor set we are looking for is

$$
C=\overline{\bigcup_{i \in \mathbb{Z}} C_{i}}=\bigcup_{i \in \mathbb{Z}} C_{i} \cup\{w\} .
$$

Note that $C$ is a Cantor set since it is perfect, compact, and totally disconnected.

Theorem 5.2. The Cantor set $C$ from the previous construction has embedding homogeneity group $\mathbb{Z}$ and is unsplittable.

Proof. It is clear from the construction that each point of $C-\{w\}$ has local genus 1 . Theorem 2.4, applied to $w$ and the Cantor sets $C_{+}=\overline{\bigcup_{i>0} C_{i}}$ and $C_{-}=\overline{\bigcup_{i<0} C_{i}}$, shows that $w$ has local genus 2 in $C$. Thus, any homeomorphism of $S^{3}$ that takes $C$ to $C$ must fix $w$.

Let $h$ be such a homeomorphism of $S^{3}$ taking $C$ to $C$. Let $T_{i}^{\prime}$ be the union of the linked tori in the Antoine chain at the second stage of the construction of $C_{i}$. Let

$$
\Lambda_{N}=\bigcup_{i=-N}^{N} T_{i}, \quad \Gamma_{N}=\bigcup_{i=-N}^{N} C_{i}, \quad \text { and } \quad \Lambda_{N}^{\prime}=\bigcup_{i=-N}^{N} T_{i}^{\prime} .
$$

Fix an integer $n \in \mathbb{Z}$. Since $h\left(T_{n}\right)$ does not contain $w$, there is a positive integer $N_{1}>|n|$ such that $h\left(C_{n}\right) \subset \Gamma_{N_{1}}$. Similarly, there is a positive integer $N_{2}>N_{1}$ such that $h^{-1}\left(\Gamma_{N_{1}}\right) \subset \Gamma_{N_{2}}$.

As in Step 1 in the proof of Theorem 3.1, there is a homeomorphism $k$ of $S^{3}$ to itself, fixed on $C$, such that

$$
k\left(h\left(\partial\left(\Lambda_{N_{2}+1}\right) \cup \partial\left(\Lambda_{N_{2}+1}^{\prime}\right)\right)\right) \cap\left(\partial\left(\Lambda_{N_{2}+1}\right) \cup \partial\left(\Lambda_{N_{2}+1}^{\prime}\right)\right)=\varnothing .
$$

Fix a point $p$ of $C_{n}$ and let $k(h(p))=h(p)=q \in C_{m}$. We will show that $k\left(h\left(C_{n}\right)\right)=$ $h\left(C_{n}\right)=C_{m}$. Let $\ell=k \circ h$. Since $\ell\left(T_{n}\right) \cap T_{m} \neq \varnothing$, and since the boundaries do 
not intersect, either $\ell\left(T_{n}\right) \subset \operatorname{Int}\left(T_{m}\right)$ or $\operatorname{Int}\left(\ell\left(T_{n}\right)\right) \supset T_{m}$. We consider these cases separately.

Case I: $\ell\left(T_{n}\right) \subset \operatorname{Int}\left(T_{m}\right)$. If $\ell\left(T_{n}\right)$ has geometric index 0 in $T_{m}$, then $\ell\left(T_{n}\right)$ is contained in a cell in $T_{m}$ and so it contracts in $T_{m}$. Since a contraction of $\ell\left(T_{n}\right)$ meets the boundary of the linked $\ell\left(T_{n+1}\right)$, and since the boundary of $\ell\left(T_{n+1}\right)$ is disjoint from the boundary of $T_{m}, \ell\left(T_{n+1}\right) \subset \operatorname{Int}\left(T_{m}\right)$. Continuing inductively, one finds that one of the following two situations occur when $\ell\left(T_{n}\right)$ has geometric index 0 in $T_{m}$ :

Case Ia: Each $\ell\left(T_{j}\right)$, for $n \leq j \leq N_{2}$, is contained in $T_{m}$ and has geometric index 0 there. It follows that $\ell\left(T_{N_{2}+1}\right) \subset \operatorname{Int}\left(T_{m}\right)$. But then $C_{m} \subset \Gamma_{N_{1}}$ and $h^{-1}\left(C_{m}\right) \cap$ $C_{N_{2}+1} \neq \varnothing$, contradicting the choice of $N_{2}$.

Case Ib: There exists $j$ with $n<j \leq N_{2}$ and such that $\ell\left(T_{j}\right)$ is contained $\operatorname{in} \operatorname{Int}\left(T_{m}\right)$ and geometric index $k$ in $T_{m}$, where $k>1$. Then, by Theorem $2.2, \ell\left(T_{j}\right)$ cannot be contained in any component of the next stage of the construction contained in $T_{m}$, since these have geometric index 0 in $T_{m}$. So some component of the next stage in $T_{m}$ is contained in $\ell\left(T_{j}\right)$ and has geometric index 0 there by Theorem 2.2. Since the components of the next stage are linked, all components of the next stage in $T_{m}$ are contained in $\ell\left(T_{j}\right)$. The geometric index of the union of the next stages of in $T_{m}$ in $\ell\left(T_{n}\right)$ is even by Theorem 2.3 and cannot be equal to 0 . Otherwise, by Theorem 2.2 the union of the next stages of $T_{m}$ would have index 0 in $T_{m}$, which is a contradiction. So the geometric index of the union of the next stages of in $T_{m}$ in $\ell\left(T_{n}\right)$ is at least 2 . Then by Theorem 2.2, the geometric index of the union of the next stages of in $T_{m}$ in $T_{m}$ is at least 4 , contradicting the fact that this geometric index is 2 .

It follows that $\ell\left(T_{j}\right)$ has geometric index 1 in $T_{m}$ and contains the union of the next stages contained in $T_{m}$. Since $\ell$ is a homeomorphism that takes $C$ to $C$, it follows from the construction of $C$ that $\ell\left(C_{j}\right)=C_{m}$. Since $\ell(p) \in C_{m}$, $\ell\left(T_{n}\right) \cap \ell\left(C_{j}\right) \neq \varnothing$, contradicting the fact that $\ell$ is a homeomorphism.

Thus, neither Case Ia nor Case Ib can occur. So the geometric index of $\ell\left(T_{n}\right)$ in $T_{m}$ must be at least 1 . Repeating the argument from Case Ib above with $T_{j}$ replaced by $T_{n}$, we see that $\ell\left(T_{n}\right)$ has geometric index 1 in $T_{m}$ and contains the union of the next stages contained in $T_{m}$. Since $\ell$ is a homeomorphism that takes $C$ to $C$, it follows from the construction of $C$ that $\ell\left(C_{n}\right)=C_{m}$ as desired.

Case II: $\operatorname{Int}\left(\ell\left(T_{n}\right)\right) \supset T_{m}$. Then $\ell^{-1}\left(T_{m}\right) \subset \operatorname{Int}\left(T_{n}\right)$. The argument from Case I can now be repeated, replacing $\ell$ by $\ell^{-1}$ and interchanging $T_{n}$ and $T_{m}$. It follows that $\ell^{-1}\left(C_{m}\right)=C_{n}$ and so $\ell\left(C_{n}\right)=C_{m}$ as desired.

Since $\ell\left(C_{n}\right)=h\left(C_{n}\right)=C_{m}$, it must be the case that $(m-n) \equiv 0 \bmod 3$. Continuing as in the proof of the $\mathbb{Z}_{m}$ result (Theorem 4.2), we have that for each $i$, 
$h\left(C_{i}\right)=C_{i+(m-n)}$. Recall that for the homeomorphism $\alpha$ from the construction of $C$, it is also the case that $\alpha^{(m-n) / 3}\left(C_{i}\right)=C_{i+(m-n)}$. By the rigidity of these Cantor sets, it follows that $\left.\alpha^{(m-n) / 3}\right|_{C_{i}}=\left.h\right|_{C_{i}}$. Thus the embedding homogeneity group of $C$ is $\left\{\alpha^{k}: k \in \mathbb{Z}\right\} \simeq \mathbb{Z}$.

We now show that $C$ is unsplittable. Assume that $\Sigma$ is a 2 -sphere in $S^{3}$ that separates $C$. Choose $\epsilon>0$ so that the distance from $\Sigma$ to $C$ is greater than $\epsilon$. Choose $N$ so that each $T_{i},|i| \geq N$, has diameter less than $\epsilon / 6$ and is within $\epsilon / 6$ of $w$. Since $\Sigma$ separates $C, w \cup \bigcup_{|i| \geq N} T_{i}$ must be in one component of $S^{3}-\Sigma$ and there must be points of $C$ in the other component of $S^{3}-\Sigma$. So $\bigcup_{|i| \leq N} T_{i}$ contains points in both components of $S^{3}-\Sigma$.

Form an Antoine Cantor set $C^{\prime}$ related to $C$ as follows. Use $\bigcup_{|i| \leq N} T_{i}$ as a part of the first stage of the construction. Complete the first stage of the construction by adding an unknotted solid torus $T$, linked to $T_{N}$ and $T_{-N}$, that is within the $\epsilon / 3$-neighborhood of $w$. For successive stages of the Antoine Cantor set $C^{\prime}$ in $T_{i},|i| \leq N$, use the successive stages in forming the Cantor set $C_{i} \subset C$. For successive stages of the Antoine Cantor set $C^{\prime}$ in $T$, use any Antoine construction.

By construction and the properties of $\Sigma$, the 2-sphere $\Sigma$ separates the Antoine Cantor set $C^{\prime}$, contradicting Remark 3.2.

\section{Main results}

Given a finitely generated abelian group $G$, we use the results from the previous two sections to construct an unsplittable Cantor set $C_{G}$ in $S^{3}$ with embedding homogeneity group $G$.

Construction 6.1. Let $G \simeq \mathbb{Z}^{n} \oplus \mathbb{Z}_{m_{1}} \oplus \mathbb{Z}_{m_{2}} \oplus \cdots \oplus \mathbb{Z}_{m_{k}}$ be any finitely generated abelian group. Form a simple chain of $n+k$ pairwise disjoint unknotted solid tori. Figure 3 illustrates the case $n+k=6$. Label the tori as $T_{1}, T_{2}, \ldots, T_{n+k}$ so that $T_{1}$ is only linked with $T_{2}, T_{n+k}$ is only linked with $T_{n+k-1}$, and each $T_{i}$, for $2 \leq i \leq n+k-1$, is linked with $T_{i-1}$ and $T_{i+1}$.

For $1 \leq i \leq n$, perform Construction 5.1 in $T_{i}$, treating a pinched version of $T_{i}$ in the interior of $T_{i}$ as the torus $S_{0}$ in Construction 5.1. Let $w_{i}$ be the limit point corresponding to $w$ in Construction 5.1. This yields a Cantor set $C_{i}$ in $T_{i}$ with embedding homogeneity group $\mathbb{Z}$. See Figure 4.

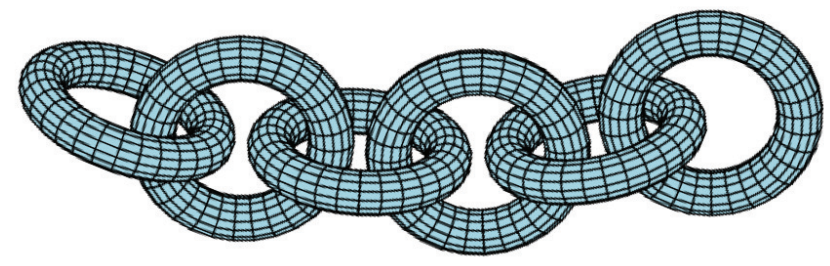

Figure 3. An Antoine chain containing $C_{G}$. 


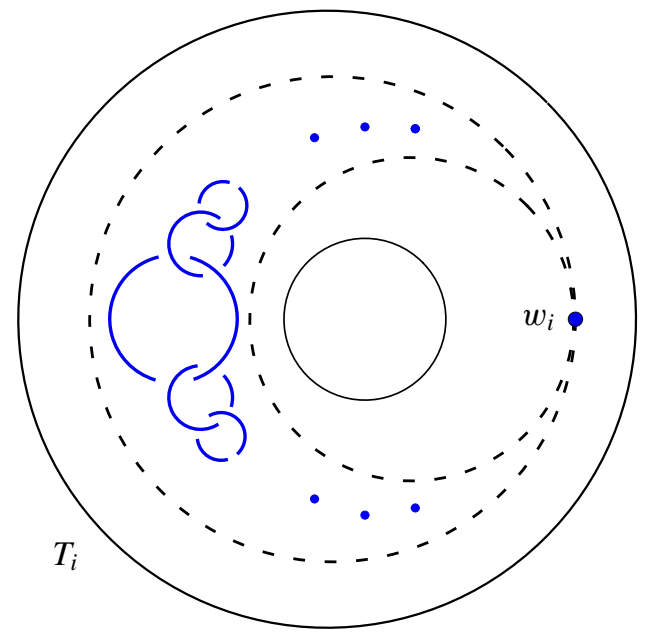

Figure 4. Pinched torus in $T_{i}$.

For $n+1 \leq i \leq n+k$, perform Construction 4.1 for the group $\mathbb{Z}_{m_{i-n}}$ in $T_{i}$. This yields a Cantor set $C_{i}$ in $T_{i}$ with embedding homogeneity group $\mathbb{Z}_{m_{i-n}}$. Choose all the rigid Cantor sets from Constructions 5.1 and 4.1 to be inequivalent.

Let

$$
C_{G}=\bigcup_{i=1}^{n+k} C_{i} .
$$

Theorem 6.2. The Cantor set $C_{G}$ constructed above has embedding homogeneity group $G$ and is unsplittable.

Proof. For $1 \leq i \leq n+k$, let $h_{i}$ be a self-homeomorphism of $S^{3}$, fixed on the complement of $T_{i}$, such that $\left.h_{i}\right|_{C_{i}}$ generates the embedding homeomorphism group of $C_{i}$ ( $\mathbb{Z}$ for $1 \leq i \leq n$ and $\mathbb{Z}_{m_{i-n}}$ for $n+1 \leq i \leq n+k$ ). Then

$$
\left\{\left.\left(h_{1}^{j_{1}} \circ h_{2}^{j_{2}} \circ \cdots \circ h_{n+k}^{j_{n+k}}\right)\right|_{C_{G}}\right\} \simeq G \simeq \mathbb{Z}^{n} \oplus \mathbb{Z}_{m_{1}} \oplus \mathbb{Z}_{m_{2}} \oplus \cdots \oplus \mathbb{Z}_{m_{k}} .
$$

Let $h$ be a homeomorphism of $S^{3}$ to itself taking $C_{G}$ to $C_{G}$. We will show that $\left.h\right|_{C_{G}}=\left.\left(h_{1}^{j_{1}} \circ h_{2}^{j_{2}} \circ \cdots \circ h_{n+k}^{j_{n+k}}\right)\right|_{C_{G}}$ for some choice of $j_{i}$.

Step 1: The homeomorphism $h$ must take each $C_{i}$ to itself. As in the proof of Theorem 5.2, there are exactly $n$ points of genus 2 in $C_{G}$, one in each $C_{i}, 1 \leq i \leq n$. These are the points $\left\{w_{1}, w_{2}, \ldots, w_{n}\right\}$. The homeomorphism must take this set of genus 2 points to itself.

Let $T$ be one of the solid torus components of the first stage of the Antoine construction for some $C_{i}, 1 \leq i \leq n+k$. As in the proofs of Theorems 4.2 and 5.2, after a general position adjustment, either $h(T)$ must lie in the interior of some solid torus component $T^{\prime}$ of the first stage of the Antoine construction for some $C_{j}$, 
or $T^{\prime}$ must lie in the interior of $h(T)$. A similar argument to that in Theorem 5.2 shows that $N\left(h(T), T^{\prime}\right)=1$ or $N\left(T^{\prime}, h(T)\right)=1$, and that $h\left(C_{i} \cap T\right)=C_{j} \cap T^{\prime}$.

This same argument can be applied to all first stage tori in $C_{i}$, resulting in the fact that $h\left(C_{i}\right)=C_{j}$. Because of the inequivalence of the rigid Cantor sets used in the construction, $i=j$ and $h\left(C_{i}\right)=C_{i}$.

Step 2: For each $i,\left.h\right|_{C_{i}}=\left.h_{i}^{k(i)}\right|_{C_{i}}$ for some $k(i)$. By Step 1, we have that $h\left(C_{i}\right)=C_{i}$. It follows from the construction that $\left.h\right|_{C_{i}}=h_{i}^{k(i)}$ for some $k(i)$. From this, it follows that $\left.h\right|_{C_{G}}=\left.\left(h_{1}^{j_{1}} \circ h_{2}^{j_{2}} \circ \cdots \circ h_{n+k}^{j_{n+k}}\right)\right|_{C_{G}}$ for some choice of $j_{i}$.

Thus, the embedding homeomorphism group of $C_{G}$ is isomorphic to $G$.

Step 3: $C_{G}$ is unsplittable. Let $\Sigma$ be a 2-sphere in $S^{3}$ separating $C_{G}$. As in the proof of Theorem 5.2, an Antoine Cantor set with first stage $\bigcup_{i=1}^{n+k} T_{i}$ can be formed so that $\Sigma$ separates this Antoine Cantor set. This is a contradiction. (See Remark 3.2.)

Corollary 6.3. Let $G \simeq \mathbb{Z}^{n} \oplus \mathbb{Z}_{m_{1}} \oplus \mathbb{Z}_{m_{2}} \oplus \cdots \oplus \mathbb{Z}_{m_{k}}$ be any finitely generated abelian group. There is a irreducible open 3-manifold $M_{G}$ with the following properties:

(a) The Freudenthal compactification of $M_{G}$ is $S^{3}$.

(b) The end set of $M_{G}$ is homeomorphic to a Cantor set.

(c) The end homogeneity group of $M_{G}$ is isomorphic to $G$.

(d) The genus of $M_{G}$ at infinity is 2 at the $n$ points corresponding to $\mathbb{Z}^{n}$ and is 1 otherwise.

Proof. Let $M_{G}$ be $S^{3}-C_{G}$, where $C_{G}$ is as in Construction 6.1. The end set of $M_{G}$ is $C_{G}$ and the end homogeneity group of $M_{G}$ is isomorphic to the embedding homogeneity group of $C_{G} . M_{G}$ is irreducible because $C_{G}$ is unsplittable. Claims (b) and (c) now follow from Theorem 6.2, while (d) follows from the proof of that theorem.

Remark 6.4. For each finitely generated abelian group $G$ as above, there are uncountably many nonhomeomorphic 3-manifolds as in the corollary. This follows from varying the rigid Cantor sets used in the construction.

\section{Questions}

Question 7.1. If a finitely generated abelian group is infinite, is there an open 3 -manifold with end homogeneity group $G$ that is genus 1 at infinity?

Question 7.2. Given a finitely generated abelian group $G$, are there simply connected open 3-manifolds with end homogeneity group $G$ ?

Question 7.3. Is the mapping class group of the open 3-manifold $M_{G}$ isomorphic to $G$ ? 
Question 7.4. If $G$ is a finitely generated nonabelian group, is there an open 3manifold with end homogeneity group $G$ ?

Question 7.5. If $G$ is a nonfinitely generated group, is there an open 3-manifold with end homogeneity group $G$ ?

\section{Acknowledgments}

The authors would like to thank the referee for a number of helpful suggestions, including a clarification of the proof of Theorem 5.2. Garity was supported in part by NSF grants DMS-0852030 and DMS-1005906. Both authors were supported in part by the Slovenian Research Agency grant BI-US/11-12-023 and BI-US/13-14-027. Repovš was supported in part by Slovenian Research Agency grants P1-0292-0101, J1-2057-0101, and J1-4144-0101.

\section{References}

[Antoine 1920] L. Antoine, "Sur la possibilité d'étendre l'homéomorphie de deux figures à leur voisinage”, C. R. Acad. Sci. (Paris) 171 (1920), 661-663. Zbl 47.0524.01

[Daverman 1979] R. J. Daverman, "Embedding phenomena based upon decomposition theory: wild Cantor sets satisfying strong homogeneity properties", Proc. Amer. Math. Soc. 75:1 (1979), 177-182. MR 80k:57031 Zbl 0407.57011

[Dickman 1968] R. F. Dickman, Jr., "Some characterizations of the Freudenthal compactification of a semicompact space", Proc. Amer. Math. Soc. 19 (1968), 631-633. MR 37 \#884 Zbl 0157.29404

[Dijkstra 2010] J. J. Dijkstra, "Homogeneity properties with isometries and Lipschitz functions", Rocky Mountain J. Math. 40:5 (2010), 1505-1525. MR 2011j:54033 Zbl 1227.54030

[Freudenthal 1942] H. Freudenthal, "Neuaufbau der Endentheorie", Ann. of Math. (2) 43 (1942), 261-279. MR 3,315a Zbl 0060.40006

[Garity and Repovš 2013] D. J. Garity and D. Repovš, "Inequivalent Cantor sets in $\mathbb{R}^{3}$ whose complements have the same fundamental group", Proc. Amer. Math. Soc. 141:8 (2013), 2901-2911. MR 3056580 Zbl 06184489

[Garity et al. 2005] D. J. Garity, D. Repovš, and M. Željko, "Uncountably many inequivalent Lipschitz homogeneous Cantor sets in $\mathbb{R}^{3}$ ", Pacific J. Math. 222:2 (2005), 287-299. MR 2006m:54056 Zbl 1116.54015

[Garity et al. 2006] D. J. Garity, D. Repovš, and M. Željko, "Rigid Cantor sets in $\mathbb{R}^{3}$ with simply connected complement”, Proc. Amer. Math. Soc. 134:8 (2006), 2447-2456. MR 2007a:54020 Zbl 1165.54309

[Garity et al. 2011] D. J. Garity, D. Repovš, D. Wright, and M. Željko, "Distinguishing BingWhitehead Cantor sets", Trans. Amer. Math. Soc. 363:2 (2011), 1007-1022. MR 2011j:54034 Zbl 1236.54025

[Garity et al. 2014] D. J. Garity, D. Repovš, and D. Wright, "Simply connected open 3-manifolds with rigid genus one ends", Revista Matemática Complutense 27:1 (2014), 291-304.

[van Mill 2011] J. van Mill, "On countable dense and strong $n$-homogeneity", Fund. Math. 214:3 (2011), 215-239. MR 2012k:54054 Zbl 1248.54016 
[Schubert 1953] H. Schubert, "Knoten und Vollringe”, Acta Math. 90 (1953), 131-286. MR 17,291d Zbl 0051.40403

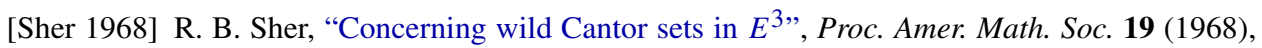
1195-1200. MR 38 \#2755 Zbl 0165.57202

[Shilepsky 1974] A. C. Shilepsky, "A rigid Cantor set in $E^{3 ",}$ Bull. Acad. Polon. Sci. Sér. Sci. Math. Astronom. Phys. 22 (1974), 223-224. MR 49 \#9849 Zbl 0275.57001

[Siebenmann 1965] L. C. Siebenmann, The obstruction to finding a boundary for an open manifold of dimension greater than five, $\mathrm{PhD}$ thesis, Princeton University, 1965, Available at http:// www.math.uchicago.edu/ shmuel/tom-readings/Siebenmann\%20thesis.pdf. MR 2615648

[Souto and Stover 2013] J. Souto and M. Stover, "A Cantor set with hyperbolic complement", Conform. Geom. Dyn. 17 (2013), 58-67. MR 3045630 arXiv 1205.4668

[Wright 1986] D. G. Wright, "Rigid sets in $E^{n ",}$ Pacific J. Math. 121:1 (1986), 245-256. MR 87b: 57011 Zbl 0586.57009

[Željko 2005] M. Željko, “Genus of a Cantor set”, Rocky Mountain J. Math. 35:1 (2005), 349-366. MR 2006e:57022 Zbl 1091.57011

Received September 11, 2012. Revised July 19, 2013.

DENNIS J. GARITY

MATHEMATICS DEPARTMENT

OREGON STATE UNIVERSITY

KIDDER HALL 368

CORVALLIS, OR 97331

UNITED STATES

garity@math.oregonstate.edu

DUŠAN REPOVŠ

FACUlty of EducAtion and FACUlty of Mathematics And Physics

UNIVERSITY OF LJUBLJANA

KARDELJEVA PLOŠČAD 16

1000 LJUBLJANA

SLOVENIA

dusan.repovs@guest.arnes.si 


\title{
PACIFIC JOURNAL OF MATHEMATICS
}

\author{
msp.org/pjm
}

Founded in 1951 by E. F. Beckenbach (1906-1982) and F. Wolf (1904-1989)

\section{EDITORS}

Don Blasius (Managing Editor)

Department of Mathematics

University of California

Los Angeles, CA 90095-1555

blasius@math.ucla.edu

\author{
Paul Balmer \\ Department of Mathematics \\ University of California \\ Los Angeles, CA 90095-1555 \\ balmer@math.ucla.edu \\ Robert Finn \\ Department of Mathematics \\ Stanford University \\ Stanford, CA 94305-2125 \\ finn@math.stanford.edu \\ Sorin Popa \\ Department of Mathematics \\ University of California \\ Los Angeles, CA 90095-1555 \\ popa@math.ucla.edu
}

\author{
Vyjayanthi Chari \\ Department of Mathematics \\ University of California \\ Riverside, CA 92521-0135 \\ chari@math.ucr.edu \\ Kefeng Liu \\ Department of Mathematics \\ University of California \\ Los Angeles, CA 90095-1555 \\ liu@math.ucla.edu \\ Jie Qing \\ Department of Mathematics \\ University of California \\ Santa Cruz, CA 95064 \\ qing@ cats.ucsc.edu
}

\section{PRODUCTION}

Silvio Levy, Scientific Editor, production@msp.org

\section{SUPPORTING INSTITUTIONS}

ACADEMIA SINICA, TAIPEI

CALIFORNIA INST. OF TECHNOLOGY

INST. DE MATEMÁTICA PURA E APLICADA

KEIO UNIVERSITY

MATH. SCIENCES RESEARCH INSTITUTE

NEW MEXICO STATE UNIV.

OREGON STATE UNIV.

\author{
STANFORD UNIVERSITY \\ UNIV. OF BRITISH COLUMBIA \\ UNIV. OF CALIFORNIA, BERKELEY \\ UNIV. OF CALIFORNIA, DAVIS \\ UNIV. OF CALIFORNIA, LOS ANGELES \\ UNIV. OF CALIFORNIA, RIVERSIDE \\ UNIV. OF CALIFORNIA, SAN DIEGO \\ UNIV. OF CALIF., SANTA BARBARA
}

\author{
Daryl Cooper \\ Department of Mathematics \\ University of California \\ Santa Barbara, CA 93106-3080 \\ cooper@math.ucsb.edu \\ Jiang-Hua Lu \\ Department of Mathematics \\ The University of Hong Kong \\ Pokfulam Rd., Hong Kong \\ jhlu@maths.hku.hk \\ Paul Yang \\ Department of Mathematics \\ Princeton University \\ Princeton NJ 08544-1000 \\ yang@math.princeton.edu
}

These supporting institutions contribute to the cost of publication of this Journal, but they are not owners or publishers and have no responsibility for its contents or policies.

See inside back cover or msp.org/pjm for submission instructions.

The subscription price for 2014 is US $\$ 410 /$ year for the electronic version, and \$535/year for print and electronic.

Subscriptions, requests for back issues and changes of subscribers address should be sent to Pacific Journal of Mathematics, P.O. Box 4163, Berkeley, CA 94704-0163, U.S.A. The Pacific Journal of Mathematics is indexed by Mathematical Reviews, Zentralblatt MATH, PASCAL CNRS Index, Referativnyi Zhurnal, Current Mathematical Publications and Web of Knowledge (Science Citation Index).

The Pacific Journal of Mathematics (ISSN 0030-8730) at the University of California, c/o Department of Mathematics, 798 Evans Hall \#3840, Berkeley, CA 94720-3840, is published twelve times a year. Periodical rate postage paid at Berkeley, CA 94704, and additional mailing offices. POSTMASTER: send address changes to Pacific Journal of Mathematics, P.O. Box 4163, Berkeley, CA 94704-0163.

PJM peer review and production are managed by EditFLOW ${ }^{\circledR}$ from Mathematical Sciences Publishers.

\section{PUBLISHED BY}

\section{mathematical sciences publishers \\ nonprofit scientific publishing}

http://msp.org/

(C) 2014 Mathematical Sciences Publishers 


\section{PACIFIC JOURNAL OF MATHEMATICS}

Volume 269 No. $1 \quad$ May 2014

The asymptotic behavior of Palais-Smale sequences on manifolds with $\quad 1$ boundary

SÉRGIO AlMARAZ

The cup subalgebra of a $\mathrm{II}_{1}$ factor given by a subfactor planar algebra is maximal amenable

ARNAUD BROTHIER

Representation theory of type B and C standard Levi $W$-algebras

JONATHAN BROWN and SIMON M. GOODWIN

New invariants for complex manifolds and rational singularities

RONG DU and YUN GAO

Homogeneity groups of ends of open 3-manifolds

DENNIS J. GARITY and DUŠAN REPOVŠ

On the concircular curvature of a $(\kappa, \mu, \nu)$-manifold

Florence Gouli-Andreou and Evaggelia Moutafi

Genuses of cluster quivers of finite mutation type

FANG LI, JICHUN LIU and YICHAO YANG

Taut foliations in knot complements

TAO LI and RACHEL ROBERTS

On the set of maximal nilpotent supports of supercuspidal representations 169

QIN YUJUN

The natural filtrations of finite-dimensional modular Lie superalgebras of 199 Witt and Hamiltonian type

Keli Zheng, Yongzheng ZHANG and WeI Song

Free Brownian motion and free convolution semigroups: multiplicative 219 case

PING ZHONG 